Article

\title{
Mean-Variance-Skewness-Entropy Measures: A Multi-Objective Approach for Portfolio Selection
}

\author{
Ilhan Usta and Yeliz Mert Kantar * \\ Department of Statistics, Science Faculty, Anadolu University, 26470 Eskisehir, Turkey; \\ E-Mail: iusta@anadolu.edu.tr
}

* Author to whom correspondence should be addressed; E-Mail: ymert@anadolu.edu.tr.

Received: 2 December 2010; in revised form: 22 December 2010 / Accepted: 29 December 2010 / Published: 12 January 2011

\begin{abstract}
In this study, we present a multi-objective approach based on a mean-varianceskewness-entropy portfolio selection model (MVSEM). In this approach, an entropy measure is added to the mean-variance-skewness model (MVSM) to generate a well-diversified portfolio. Through a variety of empirical data sets, we evaluate the performance of the MVSEM in terms of several portfolio performance measures. The obtained results show that the MVSEM performs well out-of sample relative to traditional portfolio selection models.
\end{abstract}

Keywords: portfolio selection; entropy; skewness; portfolio performance measures; out-of-sample performance

\section{Introduction}

Markowitz's mean-variance model (MVM), which is based on the assumption that returns of assets follow a normal distribution, has been accepted as a pioneer portfolio selection model [1]. It is known that the MVM depends on only the first and second moments corresponding to the expected return and the variance-covariance matrix of return. However, these moments are generally inadequate to explain portfolios in the case of non-normal return distribution [2-4]. Therefore, many studies have discussed the issue of whether higher moments should be accounted for the portfolio selection problem [2-10]. In particular, Chunhachinda et al. [2], Arditti [5] and Arditti and Levy [6] assert that higher moments cannot be neglected, unless there is a reason to believe that the asset returns are distributed normally or 
that higher moments are irrelevant to the investor's decision. Prakash et al. [4], Harvey et al. [8] and Ibbotson [10] discuss existence of the higher moments in an asset allocation system if the returns do not follow a symmetrical probability distribution. Moreover, they show that when skewness is included in the decision process, an investor can get a higher return. Due to these facts, the MVM has been extended to include the skewness of return in portfolio selection. This model is called as meanvariance-skewness model (MVSM) [2,4].

The MVM and MVSM have recently become widely-used approaches in solving portfolio diversification problems. On the other hand, some studies indicate that $[2,4,11]$ that the portfolio weights obtained from the MVM and the MVSM can often focus on a few assets or extreme positions, although an important objective of asset allocation is diversification [11,12]. In portfolio theory, it is well-known that the diversification reduces unsystematic risk in portfolios. In the other words, the more diversified portfolio weights (probabilities) there are, the more reduced risk there is in the portfolio selection $[13,14]$. Diversified portfolios also have lower idiosyncratic volatility than the individual assets [12]. Moreover, the portfolio variance decreases as the diversification in portfolio increases.

In order to measure the diversification, entropy is a widely accepted measure of diversity [15-23]. It is known that the greater the value of the entropy measure for portfolio weights, the higher the portfolio diversification is. In the literature, the first attempts to use entropy as an objective function in multi-objective model portfolio selection are seen in [19-23]. Furthermore, Bera and Park [11,19] present asset allocation models based on entropy and cross entropy measures in order to generate a well diversified portfolio. If entropy is used as an objective function to determine portfolio weights, the obtained weights become automatically non-negative. This means that a model with entropy naturally yields no short-selling, which is occasionally a preferable situation in portfolio selection due to theoretical and practical reasons [24-26].

On the other hand, the relationship between diversification and skewness has also been researched in the literature [27-30]. Several studies show that the positive skewness can lead to anti-diversification as investors attempt to capture the greatest amount of positive skewness [28]. For instance, Simkowitz and Beedles [27] examine the behavior of skewness of portfolio returns as the degree of diversification increases, and report that increasing the diversification results in a progressive loss of portfolio skewness. Besides, the results of [30] show that while the diversification reduces portfolio variance, at the same time it also reduces skewness. For this reasons, the skewness and diversification are two competing and conflicting objectives in portfolio selection.

In this study, we study multi-objective portfolio selection model in which investor tries to maximize the skewness of portfolio and entropy of portfolio weights, while simultaneously attempting to minimize the portfolio variance. Based on three different empirical datasets, we evaluate the out-of-sample performance of MVSEM relative to well-known portfolio models such as the equally weighted model (EWM), minimum variance model (MinVM), MVM and MVSM. The performances of the MVSEM are assessed in terms of the following portfolio performance measures [31-38]: the Sharpe ratio (SR), adjusted for skewness Sharpe ratio (ASR), mean absolute deviation ratio (MADR) Sortino-Satchell ratio (SSR), Farinelli-Tibiletti ratio (FTR), generalized Rachev ratio (GRR) and portfolio turnover (PT). We also compute Jobson and Korkie' $\mathrm{z}_{\mathrm{JK}}$ test statistic to evaluate the statistical significance for the difference in Sharpe ratios among the considered model in this study. 
Considering all these issues, this study is organized as follows: traditional portfolio selection models are presented briefly in Section 2. A multi-objective portfolio selection model is introduced in Section 3. The portfolio performance measures and rolling window procedure are provided in Section 4. Next, an empirical study is conducted to evaluate the performance of MVSEM in Section 5. Finally, the conclusions and suggestions are presented in Section 6.

\section{Traditional Portfolio Selection Models}

In portfolio theory, given a set of assets, the portfolio selection problem is to find the optimum way of investing a particular amount of money in these assets. Each possible strategy is considered as a portfolio selection model. In this section, we present the well-known traditional portfolio selection models and also provide definitions and notations required in this study.

The vector of portfolio weights is $\mathbf{x}=\left(x_{1}, x_{2}, \ldots, x_{n}\right)^{T}$, where $x_{i}$ is the weight of $i$ th risky asset in the portfolio. The portfolio weights satisfy $\sum_{i=1}^{n} x_{i}=x^{T} \mathbf{1}=1$, where $\mathbf{1}$ is a $n \times 1$ vector of ones and ${ }^{T}$ denotes the transpose of the vector. Additionally, the portfolio weights are constrained to be $x_{i} \in[0,1]$ $i=1, \ldots, n$, thus meaning that short selling is not allowed.

The vector of excess returns is $\mathbf{R}=\left(R_{1}, R_{2}, \ldots, R_{n}\right)^{T}=\left(\tilde{R}_{1}-r_{f}, \tilde{R}_{2}-r_{f}, \ldots, \tilde{R}_{n}-r_{f}\right)^{T}$, where $R_{i}$ represents the risk premium on the ith risky asset and $r_{f}$ is the risk-free return. The vector of mean excess returns is $E[\mathbf{R}]=\mathbf{M}=\left(m_{1}, \ldots, m_{n}\right)^{T}$, where $m_{i}=E\left(R_{i}\right)$ and $E$ denotes the expectation operator. Also, the $n \times n$ variance-covariance matrix of excess returns is $E[\mathbf{R}-E[\mathbf{R}]]^{2}=\mathbf{V}$, where $\mathbf{V}$ consists of elements of $\sigma_{i j}=E\left[\left(R_{i}-E\left[R_{i}\right]\right)\left(R_{j}-E\left[R_{j}\right]\right)\right]$, which show the covariance between the returns of asset $i$ and $j$ for $\forall(i, j) \in[1, \ldots, n]$. The $n \times n^{2}$ skewness-coskewness matrix of excess return is $E[\mathbf{R}-E[\mathbf{R}]]^{3}=\mathbf{S}$, where contains the elements of $s_{i j k}=E\left[\left(R_{i}-E\left[R_{i}\right]\right)\left(R_{j}-E\left[R_{j}\right]\right)\left(R_{k}-E\left[R_{k}\right]\right)\right]$, which represents the coskewness between the returns of asset $i, j$ and $k$ for $\forall(i, j, k) \in[1, \ldots, n]$.

Mean, variance and third central moment of the return of portfolio and the entropy of portfolio weights (probabilities) are respectively given, as follows:

$$
E\left[R_{p}\right]=E\left[\mathbf{x}^{T} \mathbf{R}\right]=\sum_{i=1}^{n} x_{i} m_{i}=\mathbf{x}^{T} \mathbf{M}
$$

where $R_{p}=\sum_{i=1}^{n} x_{i} R_{i}$ is the return of portfolio.

$$
\begin{gathered}
\sigma^{2}\left[R_{p}\right]=E\left[\mathbf{x}^{T} \mathbf{R}-E\left[\mathbf{x}^{T} \mathbf{R}\right]\right]^{2}=\sum_{i=1}^{n} \sum_{j=1}^{n} x_{i} x_{j} \sigma_{i j}=\mathbf{x}^{T} \mathbf{V} \mathbf{x}, \\
S_{3}\left[R_{p}\right]=E\left[\mathbf{x}^{T} \mathbf{R}-E\left[\mathbf{x}^{T} \mathbf{R}\right]\right]^{3}=\sum_{i=1}^{n} \sum_{j=1}^{n} \sum_{k=1}^{n} x_{i} x_{j} x_{k} S_{i j k}=\mathbf{x}^{T} \mathbf{S}(\mathbf{x} \otimes \mathbf{x}),
\end{gathered}
$$

where $\otimes$ denotes for the Kronecker product and also $S_{3}\left(R_{p}\right)$ provide a measure of skewness of portfolio $\left(S k\left[R_{p}\right]=\frac{S_{3}\left[R_{p}\right]}{\sigma_{p}^{3}\left[R_{p}\right]}\right)$. 


$$
H(\mathbf{x})=-\sum_{i=1}^{n} x_{i} \ln x_{i}=-\mathbf{x}^{T}(\ln \mathbf{x}),
$$

where $\ln \mathbf{x}$ denotes $\left(\ln x_{1}, \ldots, \ln x_{n}\right)^{T}$.

$H(\mathbf{x})$, known as Shannon's entropy measures [39], is a concave function of the portfolio weights $x_{1}, \ldots, x_{n}$. It has its maximum value $\ln n$, when $x_{i}=1 / n$ for $i=1, \ldots, n . H(\mathbf{x})$ reaches its minimum value 0 , when $x_{i}=1$ and $x_{j}=0, i \neq j$ for $j=1, \ldots, n$. Due to these properties of entropy measure, $H(\mathbf{x})$ that provides a good measure of diversity in a probability distribution, can be taken as a measure of portfolio diversification $[11,15,17]$.

\subsection{Equally Weighted Model (EWM)}

EWM considers the portfolio weights to be equal, $x_{i}=1 / n$ for $i=1, \ldots, n$, and does not involve any optimization or estimation, besides, it completely ignores the mean and variance of return. This naive rule for asset allocation has been extensively used by investors although a number of complicated derived models have been developed. Moreover, various studies in the literature such as $[11,12,40,41]$ show that the EWM works well for the out-of-sample cases.

\subsection{Minimum Variance Model (MinVM)}

In MinVM, the assets weights are obtained by minimizing only the variance-covariance matrix of the return of portfolio. MinVM can be stated as follows:

$$
\begin{gathered}
\operatorname{Min} \mathbf{x}^{T} \mathbf{V} \mathbf{x} \\
\text { subject to } \mathbf{x}^{T} \mathbf{1}=1, x_{i} \geq 0 \text { for } i=1, \ldots, n
\end{gathered}
$$

In the literature, there is empirical evidence indicating the MinVM performs better out-of-sample than MVM, even when Sharpe ratio or other performance measures, which take into account both the mean and variance, are used for the comparison [42,43].

\subsection{Mean Variance Model (MVM)}

Markowitz's MVM works by assuming that the higher expected returns can be obtained by taking more risk. MVM can be given as follows:

$$
\begin{gathered}
\operatorname{Min} \mathbf{x}^{T} \mathbf{V} \mathbf{x} \\
\text { subject to } \mathbf{x}^{T} \mathbf{M}=\mu, \mathbf{x}^{\mathrm{T}} \mathbf{1}=1 \text { and } x_{i} \geq 0 \text { for } i=1, \ldots, n
\end{gathered}
$$

where $\mu$ is the pre-determined target expected return for the portfolio.

It is known that although Markowitz's MVM is widely-used portfolio selection model, there are still some drawbacks of the MVM. For example, MVM leads to poor out-of-sample performances and the solution of MVM can often focus on a few assets or extreme positions as contrary to the notion of diversification $[11,12]$. 


\subsection{Mean Variance Skewness Model (MVSM)}

Within the framework of the MVSM, it is shown that an investor's preference for the positive skewness in the return distribution is consistent with the notion of decreasing absolute risk aversion. Also, preferences for positive skewness underline a precautionary saving motive [44]. Prakash et al. [4] emphasized that positive skewness is desirable, since increasing skewness decreases the probability of large negative values of return. The MVSM discussed in [2,4] is given in the following form:

$$
\begin{gathered}
\text { Minimize } \mathbf{x}^{T} \mathbf{V} \mathbf{x} \\
\text { Maximize } \mathbf{x}^{T} \mathbf{S}(\mathbf{x} \otimes \mathbf{x}) \\
\text { subject to } \mathbf{x}^{T} \mathbf{M}=\mu, \mathbf{x}^{T} \mathbf{1}=1 \text { and } x_{i} \geq 0 \text { for } i=1, \ldots, n
\end{gathered}
$$

In the literature, the empirical evidence related to the performance of MVSM shows that the incorporation of skewness into MVM can provide significantly better portfolios the non-normal return distributions $[2,4]$.

\section{Multi-Objective Portfolio Selection Model Based on Mean-Variance-Skewness-Entropy Measures}

The first attempts to use entropy as an objective function in portfolio analysis are seen in [11,19-23]. Among these studies, Jana et al. [21] add the entropy function to the MVSM to generate well diversified portfolios and they thus formulate the mean-variance-skewness-entropy model (MVSEM). However, they use absolute deviation instead of variance under the normality condition [32] and use a piecewise linear approximation of skewness. They also use the fuzzy programming technique to solve the multi-objective model with entropy by ignoring the evaluation of the empirical performance of the model or comparison of the model with well-known portfolio models.

In this study, we introduce the MVSEM and we also evaluate its empirical performances relative to the well-known portfolio selection models by using a variety of portfolio performance measures on different empirical data sets. The multi-objective model based on mean, variance, skewness and entropy can be expressed in the following form:

$$
\begin{aligned}
& \text { Minimize } \mathbf{x}^{T} \mathbf{V x} \\
& \text { Maximize } \mathbf{x}^{\mathrm{T}} \mathbf{S}(\mathbf{x} \otimes \mathbf{x}) \\
& \text { Maximize }-\mathbf{x}^{T} \ln (\mathbf{x}) \\
& \text { subject to } \mathbf{x}^{T} \mathbf{M}=\mu, \mathbf{x}^{T} \mathbf{1}=1 \text { and } x_{i} \geq 0 \text { for } i=1, \ldots, n
\end{aligned}
$$

To obtain the portfolio weights from MVSEM is the multi-objective optimization problem. In order to solve this problem, we use the weighted sum method (scalarization) considering its easy implementation $[45,46]$. If the weighted sum method is applied to the multi-objective optimization problem given in Equations (12)-(15), the scalarized optimization problem is obtained as follows:

$$
\begin{gathered}
\text { Minimize } \lambda_{1} \mathbf{x}^{T} \mathbf{V} \mathbf{x}-\lambda_{2} \mathbf{x}^{T} \mathbf{S}(\mathbf{x} \otimes \mathbf{x})+\lambda_{3} \mathbf{x}^{T} \ln (\mathbf{x}) \\
\text { subject to } \mathbf{x}^{T} \mathbf{M}=\mu,
\end{gathered}
$$




$$
\mathbf{x}^{T} \mathbf{1}=1 \text { and } 1>x_{i} \geq 0 \text { for } i=1, \ldots, n
$$

By assigning three weighting coefficients $\lambda_{i} \geq 0, i=1,2,3$, respectively, to each of the objective functions $\mathbf{x}^{T} \mathbf{V} \mathbf{x}, \mathbf{x}^{T} \mathbf{S}(\mathbf{x} \otimes \mathbf{x}), \mathbf{x}^{T} \ln (\mathbf{x})$, the optimal solutions of the multi-objective model can be obtained. For the computation of optimal points, the weights are chosen so as to $\lambda_{1}+\lambda_{2}+\lambda_{3}=1$. Thus, the various combination of $\lambda_{i}$ 's values represent distinct portfolio compositions. For instance, the MVSEM is identical to the MVM when $\lambda_{1}=1$ and $\lambda_{2}=\lambda_{3}=0$.

$\lambda_{i}$ can be interpreted as the risk aversion factor or risk preference of the investor for the variance, skewness of portfolio and entropy of weights, respectively. However, it should be noted that the as in most of the methods dealing with the multi-objective optimization problem, weighted sum method is essentially subjective since a decision marker has to supply the weight coefficients by taking into account the importance of each objective function within the context of the problem $[47,48]$.

\section{Portfolio Performance Evaluation}

In this section, we introduce the various portfolio performance measures and rolling window procedure to evaluate the performance of the MVSEM relative to the EWM, MinVM and MVM and MVSM.

\subsection{Portfolio Performance Measures}

In order to evaluate the performance of portfolio models, a number of alternative performance measures have been proposed in the literature [31-38]. In this study, we consider some of these performance measures. As a traditional performance measure, the Sharpe ratio (SR) has been used extensively and its formula is given as the following general form:

$$
\mathrm{SR}=\frac{E\left[R_{p}\right]}{\sqrt{\sigma^{2}\left[R_{p}\right]}},
$$

where $R_{p}$ is the return of portfolio.

However, since the SR is based on the mean-variance theory, it is only valid for normally distributed returns. Particularly, the SR can lead to misleading conclusions when the return distributions are skewed or present heavy tails [36]. Several alternatives to the SR for optimal portfolio selection have been proposed in the literature. Some of these alternatives are presented in the following:

The adjusted for skewness Sharpe ratio (ASR) [31], which takes into accounts the skewness of portfolio, is defined as follows:

$$
A S R=S R \sqrt{1+\frac{S k\left[R_{p}\right]}{3} S R}
$$

The mean absolute deviation ratio, (MADR) [32], which considers the risk as mean absolute deviation, is given as follows:

$$
\operatorname{MADR}=\frac{E\left[R_{p}\right]}{E\left[\left|R_{p}-E\left[R_{p}\right]\right|\right]} .
$$


The Sortino-Satchell ratio (SSR) and Farinelli and Tibiletti ratio (FTR) [34,35], are performance measures based on the partial moments and their formulas are given as follows, respectively:

$$
\operatorname{SSR}=\frac{E\left[R_{p}\right]}{\sqrt{E\left[\max \left(-R_{p}, 0\right)^{2}\right]}}
$$

where $E\left[\max \left(-R_{p}, 0\right)^{2}\right]$ is the lower partial moment of order 2 .

$$
\operatorname{FTR}(u ; v)=\frac{\sqrt[u]{E\left[\max \left(R_{p}, 0\right)^{u}\right]}}{\sqrt[v]{E\left[\max \left(-R_{p}, 0\right)^{v}\right]}}, \quad u, v>0,
$$

where $E\left[\max \left(-R_{p}, 0\right)^{v}\right]$ and $E\left[\max \left(R_{p}, 0\right)^{u}\right]$ are the lower partial moment of order $v$ and the upper partial moment of order $u$, respectively. The selection of $u$ and $v$ are associated to investors' styles or preferences. In the empirical part, we will consider the following cases for $u$ and $v$ according to $[33,34]: u=0.5, v=2$ for a defensive investor; $u=1.5, v=2$ for a conservative investor; $u=1, v=1$ for a moderate investor. Additionally, it is known that if $u=1, v=1$, the FTR reduces to the Omega ratio [38].

The generalized Rachev ratio (GRR) [36] is the performance measure based on the quantiles of portfolio returns and its formula is presented as follows:

$$
\operatorname{GRR}(\delta, \gamma ; \alpha, \beta)=\frac{E\left[\max \left(R_{p}, 0\right)^{\delta} \mid R_{p} \geq-\operatorname{VaR}\left(R_{p} ; 1-\alpha\right)\right]}{E\left[\max \left(-R_{p}, 0\right)^{\theta} \mid R_{p} \leq-\operatorname{VaR}\left(R_{p} ; \beta\right)\right]}, \quad \delta, \theta>0, \quad \alpha, \beta \in(0,1),
$$

where $\operatorname{VaR}\left(R_{p} ; \alpha\right)=-\inf \left\{y: P\left(R_{p} \leq y\right)>\alpha\right\}$ is the value-at-risk of $R_{p}$ at the $\alpha$ quantile level $\alpha \in(0,1)$ and $E\left[\max \left(-R_{p}, 0\right)^{\theta} \mid R_{p} \leq-\operatorname{VaR}\left(R_{p} ; \beta\right)\right]$ is $\theta$ th power expected tail loss. In the empirical part, we will use $\alpha, \beta=0.05$ as quantile levels and the values of $\delta$ and $\theta$ same as the values of $u$ and $v$, respectively in the FTR. Also, it is known that when $\delta=1, \theta=1$ in Equation (24), the GRR gives the Rachev ratio (RR) [36].

On the other hand, it should be emphasized that although there is no general agreement as to which performance measure is the best for portfolio selection in the empirical study, the measures mentioned above are all recently proposed as performance measures for assets allocation.

\subsection{Rolling Window Procedure}

In this study, the evaluation of the performance of the MVSEM relies on the rolling window procedure as described in $[11,12,42,43]$. In these procedure, firstly the sample mean, variance-covariance and skewness are estimated using an estimation window of $W=120$ or 150 monthly data. Secondly, we compute the portfolio weights according to each considered portfolio model (EWM, MinV and MVM, MVSM and MVSEM) using these sample estimates. Then, we repeat this procedure for the next period, by dropping the data for the earliest period and including the new data for the next period. We continue applying this procedure until the end of the data is reached. At the end of the procedure, we have obtained $L-W$ portfolio weight vectors for each model, where $L$ is the total number of samples in the data set. Using these portfolio weight vectors $\mathbf{x}_{t}^{T}, t=W, \ldots, L-1$, 
the out-of-sample return of portfolio in period $t+1$, denoted by $\hat{R}_{p, t+1}$, is calculated by $\hat{R}_{p, t+1}=\mathbf{x}_{t}^{T} r_{t+1}$, where $r_{t}$ denotes the return vector in period $t+1$. Thus, the outcome of this rolling-window procedure is a sequence of $L-W$ monthly out-of-sample returns generated by each of the considered portfolio models.

Based on this sequence of $L-W$ monthly out-of-sample returns, the SR, ASR, MADR, SSR, FTR and GRR measures mentioned in Section 4.1 are calculated to evaluate the performance of the MVSEM relative to EWM, MinV and MVM and MVSM. Furthermore, we consider the portfolio turnover (PT) $[12,42,43]$ as a measure of the magnitude of the transaction cost corresponding to models and we also use Jobson and Korkie' test statistic $\left(z_{J K}\right)[49,50]$ to evaluate the statistical significance for the difference in Sharpe ratios among the models considered in this study.

In line with $[12,42,43]$, the PT is defined as the average absolute change in the weights and its formula is given as follows:

$$
\mathrm{PT}=\frac{1}{L-W-1} \sum_{t=W}^{L-1} \sum_{i=1}^{n}\left|x_{i, t+1}-x_{i, t}\right|,
$$

where $x_{i, t}$ and $x_{i, t+1}$ are the portfolio weights in asset $i$ in period $t$ and $t+1$, respectively.

In order to evaluate the difference in Sharpe ratios statistically, we use the $z_{J K}$ test statistic proposed by [49] after making the correction suggested by [50]. Let $a$ and $b$ be two portfolio selection models that generates two Sharpe ratios $S R_{a}$ and $S R_{b}$, respectively. The test statistic for $S R_{a}-S R_{b}$ is asymptotically normally distributed with mean zero and variance $\vartheta$ :

$$
\vartheta=\frac{1}{L-W}\left(2-2 \rho_{a, b}+\frac{1}{2}\left(S R_{a}^{2}+S R_{b}^{2}-2 S R_{a} S R_{b} \rho_{a, b}^{2}\right)\right)
$$

where $\rho_{a, b}$ is the correlation coefficient between portfolio returns obtained from $a$ and $b$ models. Thus, the $z_{J K}$ test statistic for difference in Sharpe ratios is calculated as follows:

$$
z_{J K}=\frac{\left(S R_{a}-S R_{b}\right)}{\sqrt{\vartheta}}
$$

In this study, the $p$-value corresponding to the $z_{J K}$ test statistic will be calculated for each model with respect to the EWM, which is taken as a benchmark due to its easy implementation and widespread use. Additionally, in the literature, $[11,12,40]$ show that the EWM outperforms MVM for the out-of-sample case.

\section{The Empirical Study}

In this section, we give the descriptions of empirical datasets used in this study and present the results of the empirical study

\subsection{Data Description}

Three empirical datasets are considered in the empirical evaluation of the MVSEM. The first considered dataset consists of monthly returns on 20 industry portfolios in the United States and they are taken from Kenneth French's web site [51]. The 20 industries considered are Games, Books, 
Apparel, Chemicals, Construction, Steel, Fabricated Products, Electrical Equipment, Automotive, Carry, Telecommunications, Services, Business Equipment, Paper, Transportation, Wholesale, Retail, Meals, Finance and others. The period of dataset is from January 1993 to December 2007 (L = 180 monthly observations).

The second dataset consists of monthly seven international equity indexes, which are taken from Morgan Stanley web site [52], US, UK, Japan, Germany, France, Italy, and Canada (G-7 countries) for the period from January 1970 to September 2010 ( $\mathrm{L}=489$ monthly observations).

Last dataset includes monthly returns of 15 assets, which are traded on the Istanbul Stock Exchange (ISE) in Turkey, from different sectors: Financial Institutions, Manufacturing Industry and Technology sectors. The dataset are taken from the ISE web site [53]. The dataset period is from January 1994 to December 2007 ( $\mathrm{L}=168$ monthly observations).

It should be emphasized that all these datasets are adjusted for capital splits and stock dividends. The summary statistics for these datasets are presented in Table 1, 2 and 3, respectively.

Table 1. Descriptive statistics and normality test results for industry dataset.

\begin{tabular}{cccccc}
\hline Portfolio & Mean & Variance & Skewness & Kurtosis & JB Test \\
\hline X1 & 0.0102 & 0.0034 & -0.5559 & 1.3831 & 23.6184 \\
X2 & 0.0075 & 0.0017 & 0.1444 & 0.5086 & 2.5656 \\
X3 & 0.0071 & 0.0033 & -0.3311 & 2.4903 & 49.8034 \\
X4 & 0.0096 & 0.0023 & 0.2047 & 1.6211 & 20.9661 \\
X5 & 0.0096 & 0.0025 & -0.5567 & 1.1828 & 19.7904 \\
X6 & 0.0121 & 0.0062 & 0.1268 & 1.7839 & 24.3485 \\
X7 & 0.0127 & 0.0037 & -0.3756 & 1.0758 & 12.9134 \\
X8 & 0.0148 & 0.0033 & -0.2002 & 0.2437 & 1.6479 \\
X9 & 0.0075 & 0.0042 & -0.2532 & 0.7203 & 5.8153 \\
X10 & 0.0140 & 0.0032 & -0.7880 & 2.3812 & 61.1530 \\
X11 & 0.0064 & 0.0029 & -0.0366 & 1.5864 & 18.9161 \\
X12 & 0.0109 & 0.0050 & -0.1061 & 0.8156 & 5.3264 \\
X13 & 0.0133 & 0.0076 & -0.4505 & 1.3098 & 18.9553 \\
X14 & 0.0082 & 0.0021 & 0.0259 & 1.6327 & 20.0137 \\
X15 & 0.0084 & 0.0023 & -0.4984 & 1.3597 & 21.3170 \\
X16 & 0.0071 & 0.0019 & -0.5083 & 1.4661 & 23.8741 \\
X17 & 0.0082 & 0.0025 & -0.0993 & 0.3521 & 1.2258 \\
X18 & 0.0089 & 0.0023 & -0.4481 & 0.7969 & 10.7884 \\
X19 & 0.0113 & 0.0022 & -0.3932 & 2.8659 & 66.2398 \\
X20 & 0.0040 & 0.0026 & -0.3992 & 2.1389 & 39.0915 \\
\hline
\end{tabular}

Note: Mean, variance, skewness and kurtosis values of the returns are presented under the title of descriptive statistics; JB is value of Jarque-Bera test for normality. JB test statistic has a Chi-square distribution with two degrees of freedom. JB test has critical value of 5.99 at $5 \%$ level of significant. 
Table 2. Descriptive statistics and normality test results for international dataset.

\begin{tabular}{cccccc}
\hline Portfolio & Mean & Variance & Skewness & Kurtosis & JB Test \\
\hline X1 & 0.007 & 0.002 & -0.664 & 2.424 & 155.670 \\
X2 & 0.008 & 0.003 & -0.891 & 3.494 & 313.363 \\
X3 & 0.008 & 0.004 & -0.446 & 1.571 & 66.500 \\
X4 & 0.007 & 0.004 & -0.635 & 1.932 & 108.918 \\
X5 & 0.004 & 0.005 & -0.116 & 0.800 & 14.146 \\
X6 & 0.007 & 0.004 & -0.012 & 0.573 & 6.698 \\
X7 & 0.008 & 0.004 & 0.333 & 5.537 & 633.630 \\
\hline
\end{tabular}

Table 3. Descriptive statistics and normality test results of ISE dataset.

\begin{tabular}{cccccc}
\hline Portfolio & Mean & Variance & Skewness & Kurtosis & JB Test \\
\hline X1 & 0.043 & 0.027 & 0.262 & 1.143 & 11.059 \\
X2 & 0.031 & 0.060 & 0.227 & 1.365 & 14.482 \\
X3 & 0.037 & 0.035 & -0.455 & 2.855 & 62.845 \\
X4 & 0.041 & 0.042 & -0.077 & 0.928 & 6.191 \\
X5 & 0.040 & 0.043 & 0.354 & 2.233 & 38.410 \\
X6 & 0.035 & 0.041 & 0.261 & 1.307 & 13.875 \\
X7 & 0.036 & 0.035 & 0.081 & 1.253 & 11.181 \\
X8 & 0.038 & 0.044 & -0.258 & 3.476 & 86.450 \\
X9 & 0.040 & 0.046 & -0.367 & 1.403 & 17.551 \\
X10 & 0.033 & 0.045 & -0.055 & 1.979 & 27.492 \\
X11 & 0.038 & 0.034 & 0.152 & 2.094 & 31.333 \\
X12 & 0.023 & 0.039 & 0.263 & 1.475 & 17.156 \\
X13 & 0.036 & 0.041 & 0.690 & 2.042 & 42.549 \\
X14 & 0.025 & 0.045 & 0.794 & 3.120 & 85.785 \\
X15 & 0.029 & 0.036 & 0.562 & 1.852 & 32.857 \\
\hline
\end{tabular}

The statistics in Table 1, 2 and 3 give some insight into the characteristics of the return data. As can be seen from these tables, the Jack-Bera test for the most of return distribution of three empirical datasets reject the null hypothesis for normality at the 5\% significance level.

\subsection{Results of the Empirical Study}

In the empirical study, we choose different values of $\left(\lambda_{1}, \lambda_{2}, \lambda_{3}\right)$ in MVSEM, which can be interpreted as risk preference of investors such as $(1 / 2,0,1 / 2),(0,1 / 2,1 / 2),(2 / 4,1 / 4,1 / 4)$, $(1 / 4,2 / 4,1 / 4),(1 / 4,1 / 4,2 / 4),(1 / 3,1 / 3,1 / 3)$ as parallel in the studies $[2,4,54]$. It is known that the results of MVSEM with $(1 / 2,1 / 2,0)$ are equal to that with $(1,1,0)$ when used with the weighted sum method $[46,47]$. Therefore, in MVSEM, when $\left(\lambda_{1}, \lambda_{2}, \lambda_{3}\right)$ are taken as $(1 / 2,1 / 2,0)$, the MVSM is obtained. In other words, while equal weights are assigned to variance and skewness, entropy is weighted at zero. Likewise, the choice of $(1 / 3,1 / 3,1 / 3)$ indicates that variance, skewness and entropy are of equal importance to investors.

We evaluate empirically the performances of the MVSEM with the chosen $\left(\lambda_{1}, \lambda_{2}, \lambda_{3}\right)$ relative to the EWM, MinVM, MVM and MVSM using 20 industry portfolios, 7 international portfolios, 15 ISE 
portfolios. However, since the qualitative results regarding the MVSEM with $(2 / 4,1 / 4,1 / 4)$, $(1 / 4,2 / 4,1 / 4),(1 / 4,1 / 4,2 / 4)$ are quite similar to the MVSEM with $(1 / 3,1 / 3,1 / 3)$, we report the results only for the MVSEM with $(1 / 3,1 / 3,1 / 3)$ in this study. The results for the other values of $\left(\lambda_{1}, \lambda_{2}, \lambda_{3}\right)$ are available from authors.

All computations needed in the empirical study are conducted using the MATLAB program. It is also emphasized that the average CPU time to obtain portfolios via the MVSEM increases rapidly as the sample size and the number of assets increase due to the MVSEM's computational complexity and the long computing time needed.

For the industry dataset, we present the results for window length $\mathrm{W}=120$ in Table 4 . As seen in this table, the all considered the MVSEMs provide best results in terms of all performance measures except GRRs, which favor the EWM. Moreover, it should be noted about Table 4 that the MVM, MinVM and MVSM show the worst performance with respect to all considered performance measures. On the other hand, it is seen that the PT values of portfolios obtained from the MVSEMs are less than that of the MVM, MinVM and MVSM. This is a natural result since the resulting portfolios from MVSEMs shrink towards the equally-weighted portfolio due to entropy term. Moreover, the $p$-values for the differences in Sharpe ratios show that while the difference for the MVSEMs are not statistically significant at 5\% level, that for the MVM and MVSM is statistically significant.

Table 5 presents the results of industry dataset for window length $\mathrm{W}=150$. It can be observed that the MVSEMs performs better than MVM, MinV and MVSM according to all considered performance measures except GRR $(0.5,2)$. Taking into consideration the performance of the EWM, the EWM works better than MVSEMs according to only the SSR and GRRs. In terms of the PT, it is seen from Table 5 that the values of PT of all MVSEMs are smaller than the MVM, MinVM and MVSM.

In Tables 6 and 7, we present the results of international dataset for $\mathrm{W}=120$ and 150, respectively. Taking into account the results of Table 6 and 7 together, it is seen that the MVSEMs outperform the considered other models in terms of the most of the performance measures. Comparing the PT for the models, we observe that the values of PT for the MVSEMs are substantially less than that for the others. On the other hand, the p-values for the differences in Sharpe ratios show that none of the models yield significantly different the SR with respect to the EWM.

The results for the ISE dataset are showed in Tables 8 and 9 for window length $\mathrm{W}=120$ and 150, respectively. The obtained results for $\mathrm{W}=120$ show that the MVSEMs are able to provide a good performance relative to the EWM, MVM, MinVM and MVSM in terms of most of performance measures. Besides, for $\mathrm{W}=150$, the MVSEMs significantly outperform the other portfolio models according to all considered performance measures. Furthermore, the MVSEMs yield the lowest values of the PT for $\mathrm{W}=120$ and 150 .

Overall, we can say that portfolios obtained from the MVSEMs perform better in terms of variety portfolio performance measures than the EWM, MinVM, MVM and MVSM. Besides, the MVSEMs are able to provide smaller PT when compared to the other models. 
Table 4. The results of portfolio performance measures for industry dataset and $\mathrm{W}=120$.

\begin{tabular}{ccccccccccccc}
\hline Models & SR & ASR & MADR & SSR & FTR(0.5,2) & FTR(1.5,2) & FTR(1,1) & GRR(0.5,2) & GRR(1.5,2) & GRR(1,1) & PT & p-value \\
\hline EWM & 0.3776 & 0.3809 & 0.4672 & 1.0923 & 0.2451 & 1.4576 & 2.5817 & 114.71 & 9.6396 & 1.6717 & - \\
MinVM & 0.3157 & 0.3160 & 0.3954 & 0.8287 & 0.2015 & 1.2453 & 2.2020 & 108.64 & 7.5617 & 1.4303 & 0.1106 & 0.0524 \\
MVM & 0.3007 & 0.3005 & 0.3808 & 0.7745 & 0.1936 & 1.2026 & 2.1344 & 100.06 & 6.9410 & 1.3627 & 0.1798 & 0.0380 \\
MVSM & 0.2885 & 0.2883 & 0.3641 & 0.7348 & 0.1887 & 1.1768 & 2.0653 & 99.89 & 6.8775 & 1.3541 & 0.1693 & 0.0227 \\
$\operatorname{MVSEM}_{(1 / 2,0,1 / 2)}$ & 0.3820 & 0.3854 & 0.4763 & 1.1032 & 0.2486 & 1.4581 & 2.6123 & 110.97 & 9.3111 & 1.6435 & 0.0539 & 0.6877 \\
$\operatorname{MVSEM}_{(0,1 / 2,1 / 2)}$ & 0.3824 & 0.3858 & 0.4768 & 1.1049 & 0.2489 & 1.4590 & 2.6148 & 110.73 & 9.3038 & 1.6436 & 0.0541 & 0.7046 \\
$\operatorname{MVSEM}_{(1 / 3,1 / 3,1 / 3)}$ & 0.3822 & 0.3855 & 0.4764 & 1.1031 & 0.2486 & 1.4582 & 2.6122 & 110.98 & 9.3115 & 1.6435 & 0.0539 & 0.6873 \\
\hline
\end{tabular}

Note: The SR, ASR, MADR and SSR denote the Sharpe ratio, adjusted for skewness Sharpe ratio, mean absolute deviation ratio, Sortino-Satchell ratio, respectively. FTR(u,v) denotes the generalized Farinelli-Tibiletti ratio with different values of $u$ and $v$. $\operatorname{GRR}(\delta, \gamma)$ denotes the generalized Rachev ratio with different values of $\delta$ and $\gamma$ at quantile level $\alpha, \beta=0.05$. The PT denotes portfolio turnover. The $p$-value gives probability value corresponding to $\mathrm{z}_{\mathrm{JK}}$ test statistics of the difference between the Sharpe ratio of each models from that of the EWM benchmark. The null hypothesis of the test is that the difference between Sharpe ratios is zero.

Table 5. The results of portfolio performance measures for industry dataset and $\mathrm{W}=150$.

\begin{tabular}{ccccccccccccc}
\hline Models & SR & ASR & MADR & SSR & FTR(0.5,2) & FTR(1.5,2) & FTR(1,1) & GRR(0.5,2) & GRR(1.5,2) & GRR(1,1) & PT & p-value \\
\hline EWM & 0.3441 & 0.3381 & 0.4234 & 1.4145 & 0.0543 & 1.0790 & 2.3529 & 69.8352 & 3.7987 & 0.9413 & - & - \\
MinVM & 0.2447 & 0.2416 & 0.2975 & 0.9225 & 0.0418 & 0.9083 & 1.8224 & 67.8758 & 3.5597 & 0.9029 & 0.2370 & 0.0299 \\
MVM & 0.1819 & 0.1798 & 0.2258 & 0.6328 & 0.0373 & 0.7791 & 1.5633 & 67.7187 & 3.3612 & 0.8654 & 0.2448 & 0.0008 \\
MVSM & 0.1715 & 0.1697 & 0.2117 & 0.5948 & 0.0352 & 0.7713 & 1.5259 & 69.5960 & 3.4701 & 0.8816 & 0.1636 & 0.0005 \\
$\operatorname{MVSEM}_{(1 / 2,0,1 / 2)}$ & 0.3448 & 0.3395 & 0.4248 & 1.3693 & 0.0546 & 1.0792 & 2.3610 & 66.1523 & 3.5817 & 0.9237 & 0.0373 & 0.2721 \\
$\operatorname{MVSEM}_{(0,1 / 2,1 / 2)}$ & 0.3452 & 0.3404 & 0.4258 & 1.3737 & 0.0546 & 1.0793 & 2.3618 & 65.9892 & 3.5753 & 0.9231 & 0.0374 & 0.3005 \\
$\operatorname{MVSEM}_{(1 / 3,1 / 3,1 / 3)}$ & 0.3450 & 0.3397 & 0.4251 & 1.3692 & 0.0545 & 1.0790 & 2.3609 & 66.1638 & 3.5822 & 0.9238 & 0.0374 & 0.2718 \\
\hline
\end{tabular}


Table 6. The results of portfolio performance measures for international dataset and $\mathrm{W}=120$.

\begin{tabular}{ccccccccccccc}
\hline Models & SR & ASR & MADR & SSR & FTR(0.5,2) & FTR(1.5,2) & FTR(1,1) & GRR(0.5,2) & GRR(1.5,2) & GRR(1,1) & PT & p-value \\
\hline EWM & 0.1543 & 0.1505 & 0.2082 & 0.1248 & 1.8625 & 1.0392 & 1.5038 & 18.4701 & 1.7883 & 0.7751 & - \\
MinVM & 0.1691 & 0.1637 & 0.2266 & 0.1357 & 1.9361 & 1.0398 & 1.5588 & 19.4430 & 1.6470 & 0.7289 & 0.1199 & 0.6527 \\
MVM & 0.1495 & 0.1456 & 0.1997 & 0.1204 & 1.8745 & 1.0318 & 1.4785 & 19.4162 & 1.8105 & 0.7912 & 0.1405 & 0.3722 \\
$\operatorname{MVSM}$ & 0.1491 & 0.1452 & 0.1995 & 0.1200 & 1.8652 & 1.0296 & 1.4781 & 19.3950 & 1.7964 & 0.7864 & 0.1371 & 0.3692 \\
$\operatorname{MVSEM}_{(1 / 2,0,1 / 2)}$ & 0.1692 & 0.1657 & 0.2278 & 0.1211 & 1.8278 & 1.0407 & 1.4823 & 19.5161 & 1.8915 & 0.8122 & 0.0694 & 0.6601 \\
$\operatorname{MVSEM}_{(0,1 / 2,1 / 2)}$ & 0.1692 & 0.1656 & 0.2275 & 0.1209 & 1.8274 & 1.0407 & 1.4821 & 19.5121 & 1.8919 & 0.8123 & 0.0694 & 0.6577 \\
$\operatorname{MVSEM}_{(1 / 3,1 / 3,1 / 3)}$ & 0.1695 & 0.1659 & 0.2287 & 0.1213 & 1.8279 & 1.0414 & 1.4825 & 19.5166 & 1.8925 & 0.8123 & 0.0691 & 0.6600 \\
\hline
\end{tabular}

Table 7. The results of portfolio performance measures for international dataset and $\mathrm{W}=150$.

\begin{tabular}{ccccccccccccc}
\hline Models & SR & ASR & MADR & SSR & FTR(0.5,2) & FTR(1.5,2) & FTR(1,1) & GRR(0.5,2) & GRR(1.5,2) & GRR(1,1) & PT & p-value \\
\hline EWM & 0.1738 & 0.1688 & 0.2363 & 0.1655 & 1.2311 & 1.0132 & 1.5858 & 19.1082 & 1.8555 & 0.7980 & - & - \\
MinVM & 0.1786 & 0.1724 & 0.2422 & 0.1677 & 1.2665 & 0.9970 & 1.6000 & 19.8151 & 1.7237 & 0.7517 & 0.1063 & 0.6392 \\
MVM & 0.1696 & 0.1648 & 0.2269 & 0.1611 & 1.2429 & 1.0123 & 1.5570 & 20.1864 & 1.8797 & 0.7968 & 0.1032 & 0.3820 \\
MVSM & 0.1693 & 0.1648 & 0.2263 & 0.1615 & 1.2388 & 1.0180 & 1.5559 & 20.9188 & 1.9436 & 0.8084 & 0.1461 & 0.3729 \\
$\operatorname{MVSEM}_{(1 / 2,0,1 / 2)}$ & 0.1787 & 0.1725 & 0.2428 & 0.1698 & 1.2484 & 1.0368 & 1.5870 & 20.9453 & 1.9941 & 0.8213 & 0.0652 & 0.6456 \\
$\operatorname{MVSEM}_{(0,1 / 2,1 / 2)}$ & 0.1786 & 0.1724 & 0.2428 & 0.1697 & 1.2483 & 1.0369 & 1.5873 & 20.9437 & 1.9945 & 0.8214 & 0.0652 & 0.6449 \\
$\operatorname{MVSEM}_{(1 / 3,1 / 3,1 / 3)}$ & 0.1789 & 0.1726 & 0.2432 & 0.1701 & 1.2486 & 1.0373 & 1.5875 & 20.9460 & 1.9952 & 0.8216 & 0.0650 & 0.6455 \\
\hline
\end{tabular}


Table 8. The results of portfolio performance measures for ISE dataset and $\mathrm{W}=120$.

\begin{tabular}{ccccccccccccc}
\hline Models & SR & ASR & MADR & SSR & FTR(0.5,2) & FTR(1.5,2) & FTR(1,1) & GRR(0.5,2) & GRR(1.5,2) & GRR(1,1) & PT & p-value \\
\hline EWM & 0.3701 & 0.3605 & 0.4461 & 1.0448 & 0.1781 & 1.2098 & 2.3632 & 26.8589 & 3.6586 & 1.1685 & - & - \\
MinVM & 0.3563 & 0.3472 & 0.4286 & 0.9790 & 0.1779 & 1.1624 & 2.2576 & 31.3438 & 4.4686 & 1.2872 & 0.1704 & 0.3994 \\
MVM & 0.3892 & 0.3782 & 0.4577 & 1.1198 & 0.1797 & 1.2606 & 2.4595 & 31.9823 & 4.3659 & 1.2691 & 0.1954 & 0.6552 \\
MVSM & 0.3759 & 0.3652 & 0.4517 & 1.0613 & 0.1759 & 1.2183 & 2.3907 & 29.6981 & 3.8523 & 1.1747 & 0.2108 & 0.5471 \\
$\operatorname{MVSEM}_{(1 / 2,0,1 / 2)}$ & 0.3910 & 0.3803 & 0.4721 & 1.1310 & 0.1848 & 1.2621 & 2.5055 & 28.5031 & 3.7766 & 1.2962 & 0.0993 & 0.8928 \\
$\operatorname{MVSEM}_{(0,1 / 2,1 / 2)}$ & 0.3897 & 0.3798 & 0.4711 & 1.1293 & 0.1846 & 1.2624 & 2.5019 & 28.4221 & 3.7844 & 1.2993 & 0.0982 & 0.8762 \\
$\operatorname{MVSEM}_{(1 / 3,1 / 3,1 / 3)}$ & 0.3915 & 0.3808 & 0.4727 & 1.1321 & 0.1857 & 1.2625 & 2.5060 & 28.5150 & 3.7781 & 1.2967 & 0.0990 & 0.8829 \\
\hline
\end{tabular}

Table 9. The results of portfolio performance measures for ISE dataset and $\mathrm{W}=150$.

\begin{tabular}{ccccccccccccc}
\hline Models & SR & ASR & MADR & SSR & FTR(0.5,2) & FTR(1.5,2) & FTR(1,1) & GRR(0.5,2) & GRR(1.5,2) & GRR(1,1) & PT & p-value \\
\hline EWM & 0.3962 & 0.3940 & 0.5038 & 2.3084 & 0.0317 & 1.1254 & 2.7379 & 77.8862 & 8.0211 & 1.6044 & - & - \\
MinVM & 0.3867 & 0.3710 & 0.5191 & 1.9718 & 0.0300 & 0.9582 & 2.5791 & 24.7371 & 2.7496 & 0.9575 & 0.1761 & 0.4645 \\
MVM & 0.4740 & 0.4645 & 0.6001 & 2.9144 & 0.0343 & 1.3085 & 3.2330 & 53.0313 & 4.9134 & 1.2229 & 0.1986 & 0.8267 \\
MVSM & 0.4183 & 0.4129 & 0.5300 & 2.4419 & 0.0336 & 1.1570 & 2.7795 & 60.0778 & 5.4989 & 1.2898 & 0.1961 & 0.6347 \\
$\operatorname{MVSEM}_{(1 / 2,0,1 / 2)}$ & 0.4807 & 0.4673 & 0.6046 & 2.9134 & 0.0359 & 1.3220 & 3.2437 & 88.0680 & 9.5432 & 1.7751 & 0.1275 & 0.8477 \\
$\operatorname{MVSEM}_{(0,1 / 2,1 / 2)}$ & 0.4785 & 0.4649 & 0.6045 & 2.9239 & 0.0350 & 1.3140 & 3.2396 & 85.0866 & 9.2271 & 1.7431 & 0.1256 & 0.8308 \\
$\operatorname{MVSEM}_{(1 / 3,1 / 3,1 / 3)}$ & 0.4837 & 0.4683 & 0.6054 & 2.9421 & 0.0356 & 1.3316 & 3.2492 & 89.1761 & 9.6398 & 1.7799 & 0.1268 & 0.8466 \\
\hline
\end{tabular}




\section{Conclusions}

We present a multi-objective model which includes mean, variance, skewness of the portfolio as well as the entropy of portfolio weights and compare its performance with traditional models. This comparison is made using three different empirical datasets. As a result, we find that the performance of the MVSEM is better than the considered other models in terms of a variety of portfolio performance measures. Moreover, the MVSEM is able to provide smaller portfolio turnover in comparison to the other models, thus, it means that the transaction costs associated with the implementation of MVSEM are the lowest.

\section{References}

1. Markowitz, H. Portfolio selection. J. Finance 1952, 7, 77-91.

2. Chunhachinda, P.; Dandapani, K.; Hamid, S.; Prakash, A.J. Portfolio selection and skewness: Evidence from international stock markets. J. Bank. Finance 1997, 21, 143-167.

3. Lai, T.Y. Portfolio selection with skewness: A multiple-objective approach. Rev. Quant. Finance Acc. 1991, 1, 293-305.

4. Prakash, A.J.; Chang, C.H.; Pactwa, T.E. Selecting a portfolio with skewness: Recent evidence from US, European and Latin American equity markets. J. Bank. Finance 2003, 27, 1375-1390.

5. Arditti, F.D. Risk and the required return on equity. J. Finance 1967, 22, 19-36.

6. Arditti, F.D.; Levy, H. Portfolio efficiency analysis in three moments: The multiperiod case. J. Finance 1975, 30, 797-809.

7. Ingersoll, J. Multidimensional security pricing. J. Finan. Quant. Anal. 1975, 10, 785-798.

8. Harvey, C. R.; Liechty, J.; Liechty, M.W.; Mueller, P. Portfolio selection with higher moments; In Social Science Research Network Working Paper Series 13; Available online: http://papers.ssrn.com/sol3/papers.cfm?abstract_id=634141 (accessed on 13 December 2004).

9. Levy, H. The rationale of the mean-standard deviation analysis: Comment. Amer. Econ. Rev. 1974, 34, 434-441.

10. Ibbotson, R.G. Price performance of common stock new issues. J. Finan. Econ. 1975, 2, 235-272.

11. Bera, A.K.; Park, S.Y. Optimal portfolio diversification using the maximum entropy principle, Economet. Rev. 2008, 27, 484-512.

12. DeMiguel, V.; Garlappi, L.; Uppal, R. Optimal versus naive diversification: How inefficient is the 1/N portfolio strategy? Rev. Financ. Stud. 2009, 22, 1915-1953.

13. Dobbins, R.; Witt, S.F.; Fielding, J. Portfolio Theory and Investment Management; Blackwell Publisher: Oxford, UK, 1994.

14. Gilmore, C.G.; McManus, G.M.; Tezel, A. Portfolio allocations and the emerging equity markets of Central Europe. J. Multinat. Finan. Manage. 2005, 15, 287-300.

15. Kapur, J.N.; Kesavan, H.K. Entropy Optimization Principles with Applications; Academic Press: San Diego, CA, USA, 1992.

16. Hoskisson, R.E.; Hitt, M.A.; Johnson, R.H.; Moesel, D. Construct validity of an objective (entropy) categorical measure of diversification strategy. Strat. Manag. J. 2006, 14, 215-235. 
17. Dionísio, A.; Menezes, R.; Mendes, D.A. Uncertainty analysis in financial markets: Can entropy be a solution? In Proceedings of the 10th Annual Workshop on Economic Heterogeneous Interacting Agents (WEHIA 2005), University of Essex, Colchester, UK, 13-15 June, 2005.

18. Philippatos, G.C.; Gressis, N. Conditions of equivalence among E-V, SSD and E-H portfolio selection criteria: The case for uniform, normal and lognormal Distributions. Manag. Sci. 1975, 21, 617-625.

19. Bera, A.K.; Park, S.Y. Optimal portfolio diversification using the maximum entropy principle. In Proceedings of the Second Conference on Recent Developments in the Theory, Method, and Applications of Information and Entropy Econometrics, The American University, Washington, DC, USA, 23-25 September, 2005.

20. Usta, I.; Kantar, Y.M. Analysis of multi-objective portfolio models for the istanbul stock exchange (abstract). In Proceedings of the 2nd International Workshop on Computational and Financial Econometrics (CFE'08), Neuchatel, Switzerland, 19-21 June, 2008.

21. Jana, P.; Roy, T.K.; Mazumder S.K. Multi-objective mean-variance-skewness model for Portfolio Optimization. AMO 2007, 9, 181-193.

22. Jana, P.; Roy, T.K.; Mazumder S.K. Multi-objective possibilistic model for portfolio selection with transaction cost. J. Comput. Appl. Math. 2009, 228, 188-196.

23. Samanta, B.; Roy, T.K. Multi-objective Portfolio Optimization Model. Tamsui Oxf. J. Math. Sci. 2005, 21, 55-70.

24. Chou, P.; Li, W.; Zhou, G. Portfolio optimization under asset pricing nomalies. Jpn. World Econ. 2006, 18, 121-142.

25. Cuoco, D.; Liu, H. Optimal consumption of a divisible durable good. J. Econ. Dynam. Control 2000, 24, 561-613.

26. Shi, N.; Lai, M.; Zheng, S.; Zhang, B. Optimal algorithms and intuitive explanations for Markowitz's portfolio selection model and Sharpe's ratio with no short-selling. Sci. China Math. Phys. Astron. Technol. Sci. 2008, 51, 2033-2042.

27. Simkowitz, M.A.; Beedles W.L. Diversification in a three-moment world. J. Financ. Quant. Anal. 1978, 13, 927-941.

28. Sears, R.S.; Trennepohl, G.L. Skewness, sampling risk, and the importance of diversification. J. Econ. Bus. 1986, 38, 77-91.

29. Cromwell, N.O.; Taylor, W.R.L.; Yoder, J.A. Diversification across mutual funds in a three-moment world. App. Econ. Lett. 2000, 7, 243-245.

30. Hueng, C.J.; Yau, R. Investor preferences and portfolio selection: Is diversification an appropriate strategy? Quant. Finance 2006, 6, 255-271.

31. Zakamouline, V.; Koekebakker, S. Portfolio performance evaluation with generalized Sharpe ratios: Beyond the mean and variance. J. Bank. Financ. 2009, 33, 1242-1254.

32. Konno, H. Piecewise linear risk functions and portfolio optimization. Oper. Res. Soc. Jpn. 1990, 33, 139-156.

33. Caporin, M.; Lisi, F. Comparing and selecting performance measures for ranking assets. Marco Fanno Working Paper 2009, 0099.

34. Farinelli, S.; Ferreira, M.; Thoeny M.; Rossello D.; Tibiletti L. Optimal asset allocation aid system: From “one-size" vs. "tailor-made" performance ratio. Eur. J. Oper. Res. 2009, 192, 209-215. 
35. Farinelli, S.; Ferreira, M.; Rossello, D.; Thoeny, M.; Tibiletti, L. Beyond Sharpe ratio: Optimal asset allocation using different performance ratios. J. Bank. Financ. 2008, 32, 2057-2063.

36. Biglova, A.; Ortobelli, S.; Rachev, S.; Stoyanov, S. Different approaches to risk estimation in portfolio theory. J. Portfolio Manage. 2004, 31, 103-112.

37. Ortobelli, S.; Biglova, A.; Stoyanov, S.; Rachev, S.; Fabozzi, F. A Comparison among performance measures in portfolio theory. In Proceedings Of the 16th Ifac World Congress, Prague, Czech Republic, 4-8 July, 2005; World Congress 2005, Volume 16, Part 1.

38. Keating, C.; Shadwick, W. A universal performance measure. J. Perform. Meas. 2002, 6, 59-84.

39. Shannon, C.E. The mathematical theory of communication. Bell Syst. Tech. J. 1948, 27, 379-423. Reprinted University of Illiois Press: Urbana, IL, USA, 1998; pp. 3-91.

40. Bloomfield, T.; Leftwich, R.; Long, J. Portfolio strategies and performance. J. Financ. Econ. 1977, 5, 201-218.

41. Jorion, P. International portfolio diversification with estimation risk. J. Bus. 1985, 58, 259-278.

42. DeMiguel, V.; Nogales, F.J. Portfolio selection with robust estimation. Oper. Res. 2009, 57, 560-577.

43. Alves A.; Santos P. The out-of-sample performance of robust portfolio optimization. Braz. Rev. Financ. 2010, 8, 1-26.

44. Kimball, M.S. Precautionary saving in the small and in the large. Econometrica 1990, 58, 53-73.

45. Jahn, J. Vector Optimization; Springer: Heidelberg, Germany, 2004.

46. Kalyonmoy, D. Multi-objective Optimization Using Evolutionary Algorithms; Wiley: Chichester, UK, 2001.

47. Miettinen, K.M. Nonlinear Multiobjective Optimization; Kluwer Academic Publishers: Boston, MA, USA, 1999.

48. Deb, K. Multi-objective Optimization Using Evolutionary Algorithms. Wiley: Sussex, UK, 2001.

49. Jobson, J.D.; Korkie, R. Performance hypothesis testing with the sharpe and treynor measures. J. Finance 1981, 36, 889-908.

50. Memmel, C. Performance hypothesis testing with the Sharpe ratio. Finance Lett. 2003, 1, 21-23.

51. Kenneth French's web site. Available online: http://mba.tuck.dartmouth.edu/pages/faculty/ ken.french/ (accessed on 10 October 2010).

52. Morgan Stanley web site. Available online: http://www.morganstanley.com/about/ir/index.html (accessed on 10 October 2010).

53. ISE web site. Available online: http://www.ise.org/Data/StocksData.aspx/ (accessed on 5 May 2010).

54. Yu, L.; Wang, S.; Lai, K.K. Neural network-based mean-variance-skewness model for portfolio selection. Comput. Oper. Res. 2008, 35, 34-46.

(C) 2011 by the authors; licensee MDPI, Basel, Switzerland. This article is an open access article distributed under the terms and conditions of the Creative Commons Attribution license (http://creativecommons.org/licenses/by/3.0/). 